\title{
First Report of a Low-Grade Pseudomyxoma peritonei Originating from Gall Bladder
}

\author{
Aruna Prabhu ${ }^{a}$ Andreas Brandl ${ }^{b}$ Satoshi Wakamac Shouzou Sako d, e \\ Haruki Ishibashid $^{d-f}$ Eisei Nishino ${ }^{f}$ Yutaka Yonemurad, e, g \\ ${ }^{a}$ Department of Surgical Oncology, Thangam Cancer Center, Namakkal, India; ${ }^{\text {b }}$ Digestive Unit, Champalimaud \\ Foundation, Lisbon, Portugal; ' Department of Surgery, Graduate School of Medicine, Kyoto University, \\ Kyoto, Japan; ${ }^{\mathrm{d} D e p a r t m e n t}$ of Regional Cancer Therapy, Peritoneal Surface Malignancy Center, \\ Tokushukai Hospital, Kishiwada, Japan; ' Japanese/Asian School of Peritoneal, Surface Oncology, Osaka, Japan; \\ fDepartment of Pathology, Peritoneal Surface Malignancy Center, Tokushukai Hospital, Kishiwada, Japan; \\ gDepartment of Regional Cancer Therapy, Peritoneal Surface Malignancy Center, General Hospital, Kusatsu, Japan
}

\section{Keywords}

Cytoreductive surgery · Gallbladder cancer · Histological finding · Neoadjuvant chemotherapy · Peritoneal carcinomatosis · Pseudomyxoma peritonei

\footnotetext{
Abstract

Pseudomyxoma peritonei (PMP) refers to accumulation of mucinous ascites with or without neoplastic cells in the peritoneal cavity. It most commonly originates from a low or a high grade primary appendiceal mucinous neoplasm. Though adenocarcinoma of gall bladder has been reported to give rise to PMP, to the best of our knowledge, this is the first report of a PMP arising from a low grade mucinous tumour of the gall bladder. A 72-year-old patient was diagnosed with PMP 1.5 years after a cholecystectomy. After initial oral TS1 (combination of tegafur, gimeracil and oteracil) and later intraperitoneal (IP) chemotherapy with docetaxel and cisplatin, the patient was operated with the goal of tumour debulking, including removal of $5.5 \mathrm{~L}$ of mucinous ascites, an appendectomy, and ovariectomy. The histopathologic report showed a normal appendix and metastasis of PMP to the right ovary. After the exclusion of the 2 most common sites of origin (appendix and ovary), the specimen of the cholecystectomy was reviewed. It showed low grade mucinous tumour in the gall bladder, with immuno-histochemical markers (IHCs) suggestive of CK7, CDX2, MUC 2
}

positive and CK20, MUC5AC negative. MIB-1 index was 12\%. The pathologic report of cytoreductive surgery performed after 7 cycles of IP chemotherapy confirmed the diagnosis of PMP originating from low grade mucinous tumour of the gall bladder. Our case report illustrates a rare disease and highlights that, though peritoneal metastasis from gall bladder cancers are known to have a poor prognosis, the peritoneal dissemination from a low grade mucinous neoplasm of gall bladder (PMP) has a significantly better prognosis due to a better disease biology and improved treatment options currently available for the treatment of PMP.

C을 2020 S. Karger AG, Basel

\section{Introduction}

Pseudomyxoma peritonei (PMP) refers to accumulation of mucinous ascites with or without neoplastic cells in the peritoneal cavity. In more than $90 \%$ of the cases, the origin of PMP is a ruptured mucinous appendiceal tumour; other relatively uncommon sites are the colon, ovary, pancreas and urachus $[1,2]$.The incidence is approximately one per million per year [3]. In the past, treatment of PMP consisted of palliative debulking and chemotherapy, resulting in 10-year survival rates of $21-32 \%$ [4]. After the development of cytoreductive surgery (CRS) and hyperthermic intraperito- 

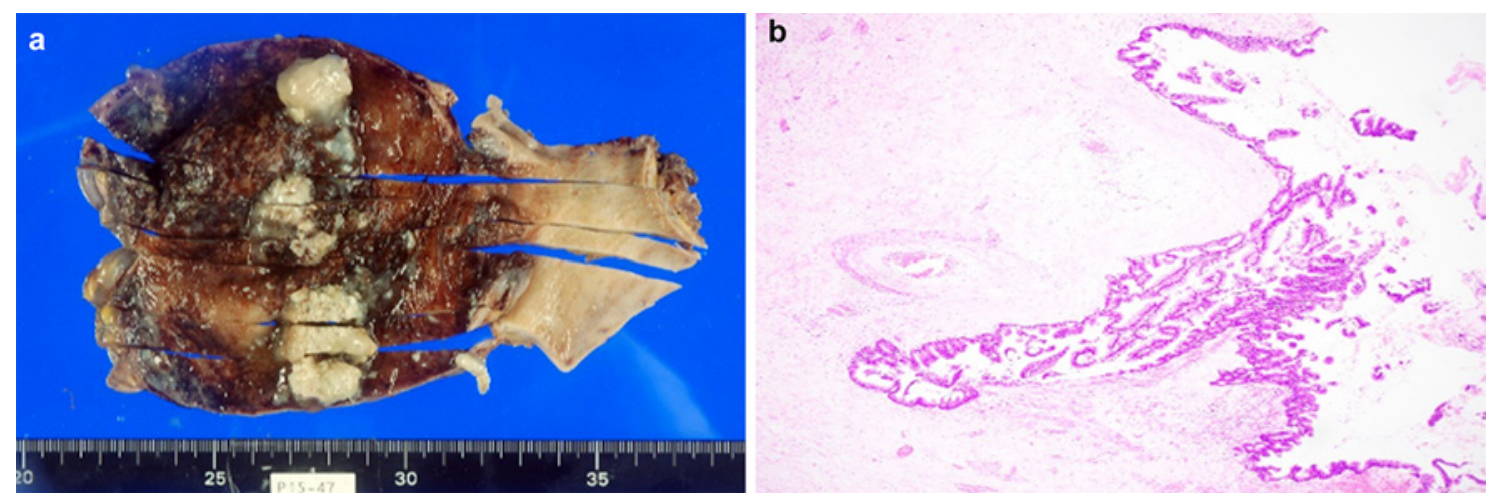

Fig. 1. Pathologic imaging of the gall bladder. a Gross specimen showing the mucinous tumour. b Microscopic examination of the primary tumour.
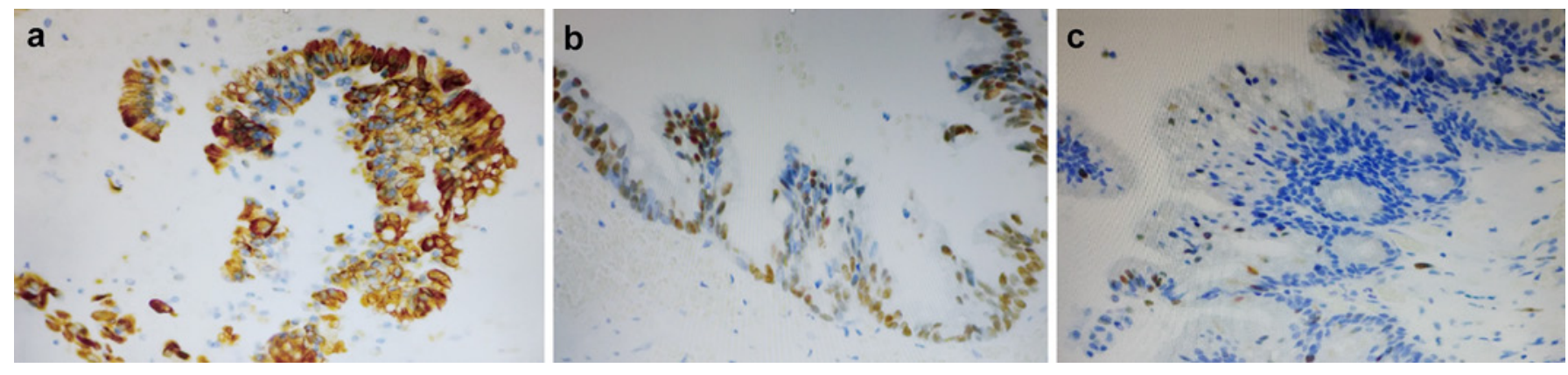

Fig. 2. Immunohistochemistry of the primary gall bladder tumour. a Expression of CK7. b Expression of CDX2. c MUC 5AC negativity.

neal chemotherapy (HIPEC), the survival of patients with PMP has considerably improved with a reported 5- and 10-year survival being 87.4 and $70.3 \%$, respectively [5].

Though adenocarcinoma of gall bladder has been reported to give rise to PMP [6], to the best of our knowledge, this is the first report of a PMP arising from a low grade mucinous tumour of the gall bladder.

\section{Case Report}

A 72-year-old fit Japanese woman presented at the local hospital with complaints of acute onset pain in the right hypochondrium with nausea and vomiting for 2 days. The patient was diagnosed to have an acute cholecystitis. Ultrasound examination showed an inflamed distended gall bladder (GB) and a distended common bile duct (CBD). She was treated with an endoscopic retrograde cholangiopancreatography (ERCP), stenting of the CBD followed by a CT scan. The CT scan revealed a suspicious thickening of GB wall, without any other signs of malignancy (no pathologically enlarged lymph nodes nor metastasis) as well as a choledochal cyst (incidental finding).

The patient was treated with a radical cholecystectomy in January 2016, including the excision of the CBD due to the choledochal cyst, reconstructed by a hepatico-jejunostomy. The postopera- tive recovery was uneventful and the patient was discharged on post-operative day (POD) 10. The histopathological report was suggestive of a high grade biliary intra-epithelial neoplasia without evidence of invasion or lymph node metastasis in the resected 6 portal lymph nodes. The patient continued on regular follow-up with clinical examination 6 monthly and annual CT scans.

Follow-up CT scan, 1 year after initial surgery, indicated mild ascites. After an observation period of 6 months, the following CT scan showed an increase of the ascites. The cytology of the ascitic fluid was reported as suspicious for peritoneal metastasis. Two of 3 serum tumour markers were elevated (CEA: $5.4 \mathrm{ng} / \mathrm{mL}$, CA19.9: $5.3 \mathrm{U} / \mathrm{mL}$ and CA125: $57.2 \mathrm{U} / \mathrm{mL}$ ). The patient was treated with oral TS1 (combination of tegafur, gimeracil and oteracil), $400 \mathrm{mg}$ twice a day. However, neither a diagnostic laparoscopy nor a biopsy were performed prior to starting TS1.

She remained asymptomatic for 1.5 years under this therapy, after which she started experiencing increasing abdominal distension. CT scan showed an increase of ascites. In view of the progressive disease, TS1 was stopped and the patient was referred to our department for further management. The repeated CT scan was suspicious for an ovarian tumour with mucinous ascites, suggestive of a PMP originating from the ovary. Tumour markers revealed an increase of CEA and CA 19.9: $13 \mathrm{ng} / \mathrm{mL}$ and $12.8 \mathrm{U} / \mathrm{mL}$, respectively. An intra-peritoneal (IP) port system was inserted under local anaesthesia and IP $40 \mathrm{mg}$ docetaxel and $40 \mathrm{mg}$ cisplatin were started on day 1 . This IP treatment was repeated 3 weekly accompanied by oral TS1 $\left(60 \mathrm{mg} / \mathrm{m}^{2}\right)$ from days 1 to 14 . After 4 cycles, in view of increasing ovarian mass with ascites, the patient 
Table 1. Immunohistochemistry panel for different primary tumours

\begin{tabular}{llllllll}
\hline \multirow{2}{*}{ Organ of origin } & \multicolumn{7}{l}{ IHC markers expressed } \\
\cline { 2 - 7 } & CK 20 & CK 7 & CDX 2 & MUC 2 & MUC5AC & MUC5B \\
\hline Appendix & + & - & + & + & + & \pm \\
Colon & + & - & \pm & + & \pm & \pm \\
Ovary & - & + & - & - & + & + \\
Pancreas & + & \pm & \pm & \pm & + & \pm \\
Urachus & + & \pm & + & + & + & NK \\
Gall bladder (in this patient) & - & + & + & + & - & NP \\
\hline
\end{tabular}

IHC, immunohistochemistry; NK, not known; NP, not performed.

was planned for an exploratory laparotomy with debulking surgery, as the patient's general condition was not fit for a CRS and HIPEC. The large $20 \times 17 \mathrm{~cm}$ right ovarian mass, including $5.5 \mathrm{~L}$ of mucinous ascites, was removed in August 2018. The appendix as well as the left ovary were removed, though grossly normal. The intraoperative peritoneal carcinomatosis index (PCI) was 30 .

The histopathological report showed a normal appendix and metastasis of PMP to the right ovary. After the exclusion of the 2 most common sites of origin (appendix and ovary), a histopathological review of the previously performed radical cholecystectomy specimen was conducted at our institute (Fig. 1, 2, Table 1). It showed low grade mucinous tumour in the GB specimen and not a non-invasive high grade biliary intra-epithelial neoplasia, as reported from the previous institute. Immuno-histochemical markers from the GB specimen as well as the peritoneal metastases were suggestive of CK7, CDX2, MUC 2 positivity and CK20, MUC5AC negativity. The MIB-1 index was $12 \%$.

An upper gastro-intestinal (UGI) endoscopy and a colonoscopy performed were normal. Hence, the diagnosis of PMP originating from low grade mucinous tumour of the GB was established. The patient was continued on IP chemotherapy and tumour markers prior to each cycle of IP chemotherapy showed a decreasing trend, indicating a response to our treatment (CEA: 2.2 ng/mL, CA: 19.9: 5.2 U/mL, CA125: $15.2 \mathrm{U} / \mathrm{mL}$ ).

After 8 cycles of IP chemotherapy, re-laparotomy was performed for CRS. The PCI was 24, $3 \mathrm{~L}$ of mucinous ascites was present. Complete cytoreduction was performed by resection of lesser and greater omentum, left sub-diaphragmatic peritoneum, resection of both sides of paracolic gutters, splenectomy and pelvic peritonectomy with preservation of rectum. The blood loss was 2,390 $\mathrm{mL}$. Following CRS, HIPEC was carried out with $1 \mathrm{~g}$ of gemcitabine for $40 \mathrm{~min}$ at $42.5-43.5^{\circ} \mathrm{C}$. Pathologic diagnosis was low grade mucinous carcinoma peritonei, and immunohistochemical study showed CK7, CDX2, MUC 2 positivity and CK20, MUC5AC negativity. The immunohistochemical study coincided with the pattern of GB epithelial cells and the primary tumour. MIB-1 index was $43 \%$. The patient was discharged on POD 20 with an uneventful postoperative course.

\section{Discussion}

The most common tumour originating from the GB is adenocarcinoma (75-80\%), with a high incidence of hematogenous, lymphatic and peritoneal metastases [7].
The other rare types of GB cancers are clear cell adenocarcinoma, signet ring cell carcinoma, squamous cell carcinoma, adeno-squamous carcinoma and undifferentiated carcinoma [8]. Most of these cases present in an advanced stage with palliative chemotherapy or best supportive care as the only treatment options.

In our patient, though there was peritoneal dissemination, the primary tumour was a low grade mucinous tumour of the GB. Although this term is not included in the 5th edition of the WHO Classification, we believe that it describes the disease most accurately. The clinical and morphologic characteristic is similar to the low grade mucinous neoplasm of the appendix, showing mild atypia in nucleus, papillary growth pattern, and stratification of the nucleus. A potential differential diagnosis is the mucinous cystic neoplasm, which is very rarely located in the gallbladder and the common bile duct, and more frequently found in the intrahepatic bile ducts and the pancreas. Also, mucinous cystic neoplasms microscopically present with cyst wall lined by flat cuboidal epithelium without atypia, overlying the ovarian type stroma, which were not present in our case.

The combination of the primary lesion, which must have been classified as a high grade biliary intraepithelial neoplasm (BillN-3, according to the WHO 2010 classification of gall bladder neoplasm), with the clinical development of PMP including spilled cancer cells from the gall bladder metastasized on the peritoneal surface by the similar metastatic pathway (superficial growing pattern) as an appendiceal mucinous neoplasm, leads to the diagnosis of PMP arising from a low grade mucinous tumour of the GB. With appropriate treatment, her prognosis is significantly better than other metastatic GB neoplasia.

Since this is the first case report of a PMP, arising from a low grade mucinous tumour of the GB, the IHC panel is unique with $\mathrm{CK} 7, \mathrm{CDX} 2$ and MUC2 positivity with $\mathrm{CK}$ 20 and MUC5AC being negative. 


\section{Conclusion}

Our case report illustrates a rare disease and highlights that, though PM from GB cancers are known to have a poor prognosis, the peritoneal dissemination from a low grade mucinous neoplasm of GB (PMP) has a significantly better prognosis due to a better disease biology and improved treatment options currently available for the treatment of PMP. Thus, a correct histopathological diagnosis is of paramount importance in choosing the appropriate treatment and counselling patients about their treatment and prognosis.

\section{Statement of Ethics}

Written informed consent was obtained from the patient for publication of this case report (including publication of images). CARE guidelines were applied for reporting this case report finding.

\section{References}

1 Pai RK, Longacre TA. Appendiceal mucinous tumors and pseudomyxoma peritonei: histologic features, diagnostic problems, and proposed classification. Adv Anat Pathol. 2005 Nov;12(6):291-311.

2 Carr NJ, Finch J, Ilesley IC, Chandrakumaran K, Mohamed F, Mirnezami A, et al. Pathology and prognosis in pseudomyxoma peritonei: a review of 274 cases. J Clin Pathol. 2012 Oct 65(10):919-23

3 Mukherjee A, Parvaiz A, Cecil TD, Moran BJ. Pseudomyxoma peritonei usually originates from the appendix: a review of the evidence. Eur J Gynaecol Oncol. 2004;25(4):411-4.

\section{Conflict of Interest Statement}

The authors have no conflicts of interest to declare.

\section{Funding Sources}

The authors have no funding sources to declare.

\section{Author Contributions}

A.P., A.B., and Y.Y. research design, performance of the research, data analysis, and paper writing. S.W. and S.S. performance of the research and data analysis. H.I. and E.N. performance of the research, data analysis, histopathological review. All authors approved the final version to be published.
4 Miner TJ, Shia J, Jaques DP, Klimstra DS, Brennan MF, Coit DG. Long-term survival following treatment of pseudomyxoma peritonei: an analysis of surgical therapy. Ann Surg. 2005 Feb;241(2):300-8.

5 Ansari N, Chandrakumaran K, Dayal S, Mohamed F, Cecil TD, Moran BJ. Cytoreductive surgery and hyperthermic intraperitoneal chemotherapy in 1000 patients with perforated appendiceal epithelial tumours. Eur J Surg Oncol. 2016 Jul;42(7):1035-41.
6 Giang TH, Ngoc TT, Hassell LA. Carcinoma involving the gallbladder: a retrospective review of 23 cases - pitfalls in diagnosis of gallbladder carcinoma. Diagn Pathol. 2012 Jan; 7(1): 10

7 Henson DE, Albores-Saavedra J, Corle D. Carcinoma of the gallbladder. Histologic types, stage of disease, grade, and survival rates. Cancer. 1992 Sep;70(6):1493-7.

8 Eken H, Balci MG, Buyukakincak S, Isik A, Firat D, Cimen O. Rare tumors of the gallbladder: clear cell carcinoma. Int J Surg Case Rep. 2015;9:65-8. 